\title{
Preparation of crosslinkable nitric oxide donor PAA/F127/PVA hybrid hydrogels for 3D printing
}

\section{Marcelo G. de Oliveira, Murilo Izidoro Santos, Mateus P. Bomediano*.}

\section{Abstract}

Nitric oxide (NO) acts in several physiological and pathophysiological processes such as wound healing and vasodilation ${ }^{1}$, which has stimulated the development of hydrogels capable of releasing NO locally in biomedical applications. The main objective of this project is the development of photocrosslinkable NO donor hybrid hydrogels for the 3D printing of biomaterials. The hydrogels were synthesized from the photopolymerization of acrylic acid in the presence of poly (ethylene oxide)-poly (propylene oxide)-poly (ethylene oxide) (PEO-PPO-PEO-F127) triblock copolymer and poly (vinyl alcohol) (PVA). To explore the ability of these hydrogels for specific applications such as cartilage replacement we used a DLP 3D printer. The characterization of the physico-chemical properties of the hydrogels included swelling, mechanical tensile and compression tests and morphology by scanning electron microscopy. The hydrogels were charged with the NO donor S-nitrosoglutathione (GSNO) and the kinetics of real-time NO release under physiological conditions was characterized by chemiluminescence.

\section{Key words:}

biomaterials, 3D printing, nitric oxide.

\section{Introduction}

Hydrogels are polymeric networks that have been widely used in medical applications due to their similarity to living tissues $^{2}$. The biomedical potential of hydrogels can be improved by incorporating active principles that promote the healing of lesions, such as, nitric oxide (NO) donors ${ }^{3}$. Photopolymerizable hydrogels can be molded by 3D printing, allowing the possibility of integration with software capable of that reproducing pieces according to the need of shape and size.

This project focuses on the development of a 3D printable hydrogel, with mechanical properties adequate for the replacement of cartilaginous tissues, capable of releasing NO locally.

\section{Results and Discussion}

Hybrid poly(acrylic acid) (PAA)/Pluronic F127 (F127)/poly(vinyl alcohol) (PVA) hydrogels were obtained in the absence of PVA and with a reference PAA/F127/PVA composition of $30 \% / 20 \% / 1 \%(w / v)$, respectively. Changes in the relative amount of $\mathrm{F} 127$ and PVA were shown to lead to different swelling degrees and to significantly affect the mechanical properties. The presence of PVA led to a significant increase in the tensile and compression properties. S-nitrosothiol (GSNO) impregnation led to hybrid PAA/F127/PVA/GSNO hydrogels capable of releasing $\mathrm{NO}$ spontaneously in physiological conditions. The 3D printing feasibility was confirmed by 3D/DLP printing.

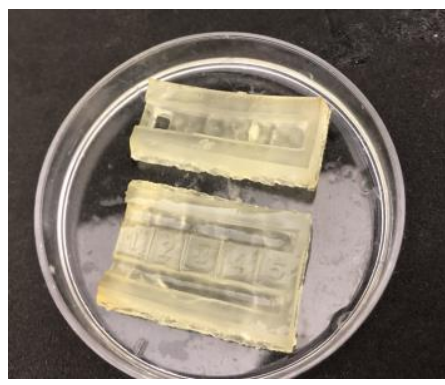

Image 1. Representative 3D-printed calibration piece of PAA/F127/PVA hydrogel.

\section{Conclusions}

PAA/F127/PVA hydrogels can be obtained by DLP/3D printing with controllable mechanical properties. PAA/F127/PVA/GSNO hydrogels release NO spontaneously and have potential for biomedical applications.

\section{Acknowledgement}

The authors acknowledge FAPESP (projects 2016/02414-5 and 2019/07325-9) for the financial support.

\footnotetext{
${ }^{1}$ Michele C. Jen, M. C.; Serrano, M. C.;van Lith, R.; Ameer, G. A. PolymerBased Nitric Oxide Therapies: Recent Insights for Biomedical Applications. Advanced Functional Materials. 2012, 22(2), 239-260.

${ }^{2}$ A. S. Hoffman. Hydrogel for biomedical applications. Adv Drug Deliv Rev, 2002, 43, 3-12.

3 de Oliveira MG. S-Nitrosothiols as Platforms for Topical Nitric Oxide Delivery. Basic \& Clinical Pharmacology \& Toxicology. 2016, 119, 49-56.
} 